\title{
Correction to: Is Pre-season Eccentric Strength Testing During the Nordic Hamstring Exercise Associated with Future Hamstring Strain Injury? A Systematic Review and Meta-analysis
}

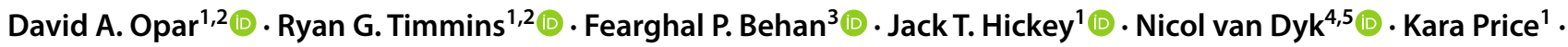 \\ Nirav Maniar ${ }^{1} \mathbb{C}$
}

Published online: 8 May 2021

(c) Springer Nature Switzerland AG 2021

\section{Correction to: Sports Medicine}

https://doi.org/10.1007/s40279-021-01474-1

The middle name of the authors are abbreviated, the correct authors names are copied below.

- Ryan Gregory Timmins—should be Ryan G. Timmins.

- David Andrew Opar—should be David A. Opar.

- Fearghal Patrick Behan—should be Fearghal P. Behan.

- Jack Thomas Hickey—should be Jack T. Hickey.

The original article has been corrected.

The original article can be found online at https://doi.org/10.1007/ s40279-021-01474-1.

David A. Opar

David.opar@acu.edu.au

1 School of Behavioural and Health Sciences, Australian

Catholic University, Fitzroy, VIC, Australia

2 Sports Performance, Recovery, Injury and New Technologies (SPRINT) Research Centre, Australian Catholic University, Fitzroy, VIC, Australia

3 Musculoskeletal Mechanics Group, Imperial College London, London, UK

4 High Performance Unit, Irish Rugby Football Union, Dublin, Ireland

5 Aspetar Orthopaedic and Sports Medicine Hospital, Doha, Qatar 